Hautarzt 2010 $61: 193-194$

DOI 10.1007/s00105-010-1929-4

๑) Springer-Verlag 2010

\author{
C. Ulrich ${ }^{1} \cdot$ K. Schäkel ${ }^{2} \cdot$ E. Stockfleth ${ }^{1}$ \\ ${ }^{1}$ Hauttumor Centrum, Charité-Universitätsmedizin \\ Berlin, Campus Virchow-Klinikum, Berlin \\ ${ }^{2}$ Universitäts-Hautklinik, Heidelberg
}

\title{
Hauterkrankungen nach Organtransplantation
}

Nach Jahren intensiver immunologischer Forschungstätigkeit stießen Murray et al. mit der ersten erfolgreichen Nierentransplantation zwischen eineiigen Zwillingen 1954 das Tor zu einem der faszinierendsten Bereiche der modernen Medizin auf. Oftmals unbekannt ist allerdings, dass die Wurzeln der Transplantationsmedizin im Bereich der Dermatologie lagen. Inspiriert durch die dermatochirurgische Versorgung von Verbrennungspatienten des Zweiten Weltkrieges, verfolgte der britische Wissenschaftler Peter Medawar den unterschiedlichen Verlauf von Hauttransplantaten, die entweder vom Patienten selbst (Autograft) oder von einem fremden Spender (Allograft) gewonnen wurden. Dabei untersuchte er die Klinik der Abstoßungsreaktionen und bereitete den Weg zu einem umfassenden Verständnis der Transplantationsimmunologie.

Während in der Frühphase der Transplantationsmedizin Patienten noch mittels Ganzkörperbestrahlung „immunsupprimiert“ wurden, konnte durch die Entdeckung wirkungsvoller systemischer Immunsuppressiva wie Azathioprine (1962) und besonders Cyclosporin (1972) die moderne Transplantationsmedizin als lebensrettende und lebensverlängernde Therapieoption an vielen Transplantationszentren weltweit etabliert werden. Heutzutage leben weltweit geschätzte 1.000.0oo organtransplantierte Patienten. Viele technische und medizinische Herausforderungen der Anfangszeit wurden inzwischen gelöst. So konnte von 1988 auf 1996 die durchschnittliche Transplantatüberlebenszeit von 7,9 auf 13,8 bei sog. „Leichenspenden“ und von 16,9 auf 35,9 bei Lebendspendern in den USA annähernd verdoppelt werden [1].

\section{Hauttumorepidemie bei Immunsupprimierten als Herausforderung für die Dermatologie des 21. Jahrhunderts}

Als neue, zentrale Herausforderung des langfristigen Überlebens nach Transplantation entwickelten sich die Folgen einer chronischen und auch derzeit noch weitgehend unselektiven Immunsuppression. Die zur Prävention von Abstoßungsreaktionen eingesetzten Immunsuppressiva inhibieren maßgeblich die zelluläre Immunüberwachung und sorgen - gerade an der Haut - für eine dramatische Zunahme von Neoplasien. Nach Untersuchungen an über 35.000 Nierentransplantierten in den USA lag die kumulative Inzidenz nichtmelanozytärer Hauttumoren nach 3 Jahren bei über $7 \%$. Verglichen mit der immunkompetenten Allgemeinbevölkerung errechnete sich daraus eine Steigerung des Hautkrebsrisikos um den Faktor 20 [2]. Hauttumore und hier besonders invasive Plattenepithelkarzinome (PEC) sind die mit Abstand am häufigsten diagnostizierten Malignome bei Organtransplantierten. Durch ihr multifokales Auftreten sowie ein ungleich aggressiveres Wachstum stellen post transplantationem auftretende PEC eine vitale Bedrohung für immunsupprimierte Patienten dar. Klassische dermatochirurgische Therapieformen als alleiniger Behandlungsansatz sind nicht in der Lage, auch nur mittelfristig den beginnenden Circulus vitiosus aus immer rascher aufeinanderfolgenden De-novo-PEC zu durchbrechen. Seitens der Transplantationsmedizin wird versucht, diesem Trend durch eine maßgeschneiderte Immunsuppression entgegenzuwirken. Beispielhaft sei die Entwicklung neuartiger Immunsuppressiva aus der Gruppe der mTOR-Inhibitoren genannt. mTOR-Inhibitoren stellen einerseits vollwertige Immunsuppressiva dar, korrelieren jedoch durch ihren Anti-VEGF-Effekt und eine ergänzende, proliferationshemmende Wirkung in klinischen Studien und Tiermodellen negativ mit der Entwicklung von Plattenepithelkarzinomen.

Epidemiologische Analysen bekannter dermatologischer Risikofaktoren für die Hauttumorgenese bei Organtransplantierten erlauben mittlerweile eine individuell adaptierte Nachsorge der Patienten [3]. Inspiriert und unterstützt durch einen entsprechenden Arbeitskreis der Arbeitsgemeinschaft Dermato-Onkologie (ADO) wurden dermatologische Nachsorgeprogramme zwischenzeitlich an vielen interdisziplinären Transplantationszentren aufgebaut. Sie ermöglichen durch umfassende dermatologische ScreeningUntersuchungen eine frühzeitige primärund sekundärprophylaktische Versorgung dieser Hochrisikogruppen und belegen die wachsende Bedeutung der Dermatologie im Kontext der modernen Transplantationsmedizin $[4,5]$.

Das vorliegende Leitthemenheft soll einen Überblick über Hintergründe und aktuelle Konzepte zur dermatologischen Versorgung von Organtransplantierten geben. Gleichzeitig soll es Klinikärzte, Wissenschaftler und explizit auch niedergelassene Kolleginnen und Kollegen ermuntern, sich mit Hautinfektionen und Hauttumoren bei Immunsupprimierten proaktiv zu befassen und entsprechende 
Strukturen und Netzwerke in Österreich, der Schweiz und Deutschland zu unterstützen.

Ohne die hervorragenden Beiträge unserer Autoren wäre ein solches Leitthemenheft nicht möglich gewesen - ihnen sei an dieser Stelle ganz herzlich für ihre Mitarbeit gedankt!

\section{1h. Smitul}

Prof. Dr. K. Schäkel

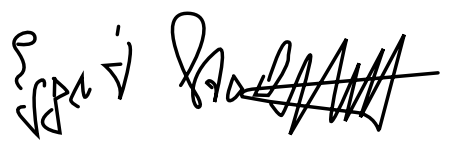

Prof. Dr. E. Stockfleth

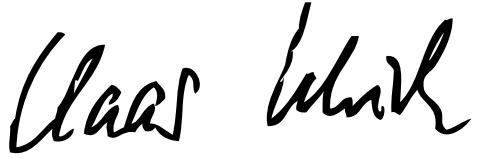

Dr. C. Ulrich

\section{Korrespondenzadresse \\ Dr. C. Ulrich}

Hauttumor Centrum, Charité-Universitätsmedizin Berlin, Campus Virchow-Klinikum Augustenburger Platz 1, 13353 Berlin claas.ulrich@charite.de

\section{Literatur}

1. Hariharan S, Johnson CP, Bresnahan BA et al (2000) Improved graft survival after renal transplantation in the United States, 1988 to 1996. N Engl J Med 342:605-612

2. Kasiske BL, Snyder JJ, Gilbertson DT et al (2004) Cancer after kidney transplantation in the United States. Am J Transplant 4:905-913

3. Ulrich C, Kanitakis J, Stockfleth E et al (2008) Skin cancer in organ transplant recipients - where do we stand today? Am J Transplant 8:2192-2198

4. Ismail F, Mitchell L, Casabonne D et al (2006) Specialist dermatology clinics for organ transplant recipients significantly improve compliance with photoprotection and levels of skin cancer awareness. Br J Dermatol 155:916-925

5. Kidney Disease: Improving Global Outcomes (KDIGO) Transplant Work Group (2009) KDIGO clinical practice guideline for the care of kidney transplant recipients. Am J Transplant 9(Suppl 3):S1-155

\section{Den wissenschaftlichen Nach- wuchs fördern: Die DGA schreibt wissenschaftliche Preise aus}

Die Deutsche Gesellschaft für Angiologie Gesellschaft für Gefäßmedizin e.V. (DGA) vergibt 2010 drei wissenschaftliche Preise: Den Promotionspreis, den Schwarz-Monheim-Preis und den Max-Ratschow-Preis. Junge Wissenschaftler sind herzlich eingeladen, sich zu bewerben und ihre Arbeiten einzureichen.

\section{Promotionspreis}

Der Preis ist mit 2.500 Euro dotiert. Der Preisträger erhält ferner eine dreijährige kostenlose Mitgliedschaft in der DGA inklusive des Bezuges der Fachzeitschrift VASA-European Journal of Vascular Medicine.

Der Preis wird für eine Dissertation, die an einer deutschen Universität abgeschlossen wurde und sich mit der klinischen und experimentellen Angiologie und ihren Grenzgebieten befasst, vergeben. Bewerben können sich die Promovierten oder die Hochschullehrer, die die Arbeit betreut haben. Zur Teilnahme sind alle zugelassen, deren Promotionsverfahren innerhalb der letzten zwei Jahre abgeschlossen wurde. Die Bewerbungen sind an den Präsidenten der DGA, Prof. Karl-Ludwig Schulte, Gefäßzentrum Berlin,

zu richten.

Es werden drei Exemplare und eine Kopie der Promotionsurkunde erbeten.

Bewerbungsfrist ist der 30. Juni 2010

\section{Schwarz-Monheim-Preis}

Der jährlich vergebene Schwarz-MonheimPreis ist mit 10.000 Euro dotiert. Er wird für abgeschlossene Forschungsarbeiten auf dem Gebiet der klinischen und experimentellen Angiologie vergeben. Die Arbeiten sollten im Jahr vor der Bewerbung in anerkannten deutsch- oder englischsprachigen Fachzeitschriften publiziert oder zur Publikation angenommen worden sein.

Bewerben können sich Erstautoren bis 40 Jahre aus einem deutschsprachigen Land. Die Bewerbungen sind in dreifacher Ausführung an den Präsidenten der Deutschen Gesellschaft für Angiologie,
Prof. Karl-Ludwig Schulte, Gefäßzentrum Berlin, Ev. Krankenhaus Königin-ElisabethHerzberge, Herzbergstr. 79, 10362 Berlin zu richten.

Bewerbungsfrist ist der 30. Juni 2010

\section{Max-Ratschow-Preis}

Alle zwei Jahre vergibt die DGA den MaxRatschow-Preis für hervorragende Arbeiten auf dem Gebiet der klinischen Angiologie. Er ist mit 5.000 Euro dotiert. Die Arbeit muss zur Veröffentlichung angenommen oder bereits veröffentlicht sein. Bewerbungen sind in dreifacher Ausführung an den Präsidenten der Deutschen Gesellschaft für Angiologie, Prof. Karl-Ludwig Schulte, Gefäßzentrum Berlin, Ev. Krankenhaus Königin-Elisabeth-Herzberge, Herzbergstr. 79, 10362 Berlin zu richten.

Die Preisträger werden im Rahmen der 39. Jahrestagung der DGA auf der diesjährigen Dreiländertagung mit den Schweizerischen und Österreichischen Gesellschaften für Angiologie vom

12. bis 15. September 2010 in Basel geehrt.

Details zu den Ausschreibungen: http://www.dga-gefaessmedizin.de/ Ausschreibungen-2010.88.0.html 